\title{
Simulation Modeling of Route Guidance Concept
}

\author{
U. VANDEBONA AND P. K. UPADHYAY
}

\begin{abstract}
The methodology of a simulation model developed at the University of New South Wales, Australia, for the evaluation of performance of Dynamic Route Guidance Systems (DRGS) is described. The microscopic simulation model adopts the event update simulation method and allows assessment of route guidance performance under different scenarios such as varying levels of participation in guidance technologies and different intensities of travel demand. This research is important for marketing, costing, and introducing route guidance and can assist planners in identifying suitable networks and traffic conditions for such systems. The example application investigates selected operating scenarios of intersection delay conditions under different compositions of participation rates in route guidance. Preliminary investigations indicate that the effectiveness of route guidance is related to the level of intersection delays. An interesting outcome of this application is the identification that in networks with intersection delays which vary with time, those motorists without guidance assistance strive to make better forecasts of travel time in order to assist their route selection. At low levels of intersection delays, some motorists appear to make more than one nonoptimal route selection.
\end{abstract}

The development of Dynamic Route Guidance Systems (DRGS) that incorporate advances in information technology to distribute the traffic efficiently over a network is a growing area of research interest. It is anticipated that the concept of DRGS will provide costeffective solutions for congestion management. This research team has been investigating methods to quantify and analyze the benefits from a generic DRGS proposal.

The importance of DRGS in urban road networks is examined. The proposed evaluation model designed to appraise projects about route guidance is explained. The evaluation methodology allows quantification of travel time savings delivered to various types of motorists in the traffic stream. The model contains four sub-models and adopts the event update simulation method to investigate the effects of dynamic route guidance. A description of the component models is reported.

To analyze the performance of DRGS, benefits of route guidance have been assessed under different scenarios such as different levels of subscription, intensities of travel demand, and sizes as well as configurations of networks. These evaluations are useful in estimating optimum levels of subscription for such systems for successful congestion management. Impacts of DRGS on the route selection behavior of different types of motorists can also be studied using this simulation model.

In the model application reported here, the evaluation model has been applied to investigate the effects of turning delays at intersections. Impacts of DRGS have been investigated under three scenarios related to intersection delay conditions.

School of Civil Engineering, University of New South Wales, Sydney NSW 2052, Australia.

\begin{abstract}
NEED FACTORS
Despite efforts to improve urban and interurban traffic conditions, congestion is still pervasive in many of these areas worldwide. As a result of advancements in information technology in the past decade, transport researchers have recognized the applicability of DRGS to tackle traffic congestion.
\end{abstract}

\section{Need for Dynamic Route Guidance Systems}

Research has shown that the potential benefits in terms of travel cost savings can justify the concept of DRGS (1). In urban road networks, motorists often travel longer routes because they are unfamiliar with alternatives. This phenomenon is relatively frequent in large road networks and leads to excess travel time and distance. Estimates indicate excess distance traveled in the United Kingdom, for 1977 to be about 6 billion $\mathrm{km}$ (3.72 billion mi) (2). This excess was generally due to drivers' inability to select the quickest routes. The economic value of this excess travel is estimated at around 540 million sterling pounds. Estimates for 1985 are $£ 2.5$ billion (3). Similar estimates for excess travel in the United States for 1983 accounted for 135 billion $\mathrm{km}$ ( 83.7 billion $\mathrm{mi})$ with an estimated cost of $\$ 45$ billion (4). These studies show that excess travel contributes to a substantial waste of transportation resources that is rapidly increasing.

Excess travel in urban road networks occurs primarily because of the limited number of route choices considered by motorists and their inaccurate perceptions of traffic conditions. Performance of road networks can be improved by advising motorists of optimum routes.

DRGS attempts to automate the acquisition, processing, and transmission of performance data to assist motorists in selecting the quickest route for a particular origin-destination pair at a given time of day. Typically, DRGS consists of control center vehicles supported by historical and real-time traffic information about the road network, along with on-board electronic devices that can communicate with control centers. These vehicles are often called "intelligent vehicles." In this instance, such traffic is referred to as "guided motorists." The objective of dynamic route guidance is to identify optimum routes for guided motorists and to navigate them through their journey.

Apart from reducing excess travel, route guidance also contributes to road safety. Research on road safety aspects reveals that such a decision-support system reduces the workload on drivers and reduces chances of such drivers causing incidents (5). It has been suggested that route selection and navigational assistance provided by DRGS reduce the mental effort required by drivers to a level similar to that experienced when driving on familiar routes. Moreover, 
reduction in excess travel has other dividends such as savings on petroleum consumption, reduced level of air and noise pollution, as well as decrease in wear and tear of the road system.

As a result of technological advancements in the transport sector, a number of DRGS are operational in the United States, Europe, and Asia (6). Since these projects involve significant investments, it is essential to assess the feasibility and suitability of such systems prior to implementation.

\section{Need for Evaluation Models}

Transport scientists involved in the development and implementation of DRGS are often challenged by the question of cost-effectiveness of such systems. Responding to these challenges requires research that is based not only on the system performance measures but also on market research. Questions such as Who will use these systems? How often? For what trip purposes? and In what situations? are basic components related to effectiveness evaluation. The following factors also need to be considered in assessing benefits of DRGS:

- Traffic authorities and technological entrepreneurs want to know the amount of benefits available from route guidance in terms of overall reductions in travel cost, traffic congestion, and air and noise pollution, and improvements in travel safety. Moreover, as funding agencies they are interested in knowing the market potential of route guidance before committing their financial resources.

- Individual drivers who are the end users of guidance systems would like to know the personal decreased travel costs in addition to increased comfort and convenience if they decide to subscribe to such guidance systems.

According to the literature, various evaluation models have been developed with the aim of estimating the potential of savings in travel time. In general, the evaluation models for DRGS adopt either an analytical approach or simulation method for measuring the performance and effects on behavior of motorists. A comparison of available assessment models related to DRGS has been reported elsewhere (6).

\section{MODEL STRUCTURE}

The proposed model for performance evaluation of DRGS is based on the estimation of the possible travel time savings from simulation of the route guidance system. The evaluation model is based on the typical event update simulation technique and treats vehicles on an individual basis. Figure 1 shows the structure and interrelationship among submodels of the DRGS model. The model consists of four components: vehicle generation, route selection, vehicle progression, and analysis.

\section{Input Sub-Model}

The input data for the model primarily consists of three data sets: network data, travel demand data, and user-defined simulation details. Network data includes the information about intersections and roads in the network. Travel demand data describes the flow of motorists between pairs of origins and destinations. User-defined simulation details include duration of simulation, proportion of different types of motorists, and description of input and output files.



FIGURE 1 Structure of simulation model.

\section{Vehicle Generation Submodel}

The vehicle generation submodel creates vehicles at regular intervals during the simulation. Each generated vehicle is attributed with a vehicle identification, motorist type, journey commencing time, origin, destination, and route. Using a pseudo-random generator, the model adopts a probabilistic method for assigning motorist type (guided or unguided) to each generated vehicle. An origin-destination pair is allocated according to stochastic methods to match the current travel demand between each origin-destination pair.

\section{Route Selection Submodel}

The route selection submodel evaluates the route between the origin and the destination of a vehicle, according to the appropriate route selection method for that particular type of vehicle. The model caters to two categories of motorists referred to as guided and unguided motorists. Guided motorists follow the routes suggested to them by the advisory system. Unguided motorists cannot access the route guidance advice and, therefore, select their travel routes by applying their prior knowledge about the road network. Unguided motorists have been further classified as passive and active. Active motorists are able to modify their routes depending on prevailing traffic conditions on the way to their destination, whereas passive motorists have no such flexibility and always adhere to their initially chosen routes. Active unguided motorists represent the category of motorists who are familiar with the locality and are able to assess when a change in a route will be beneficial based on traffic conditions they face. Procedures involved in the route selection for unguided and guided motorists are shown in Figure 2.

\section{Routes of Passive Unguided Motorists}

It is assumed that all unguided motorists have used minimum travel time routes, i.e., quickest routes. The research on various route choice models indicates that a majority of motorists attempt to travel on quickest routes. Some motorists, however, do not actually travel on such routes although they believe they have been traveling on the quickest routes. This happens because of distribution of 


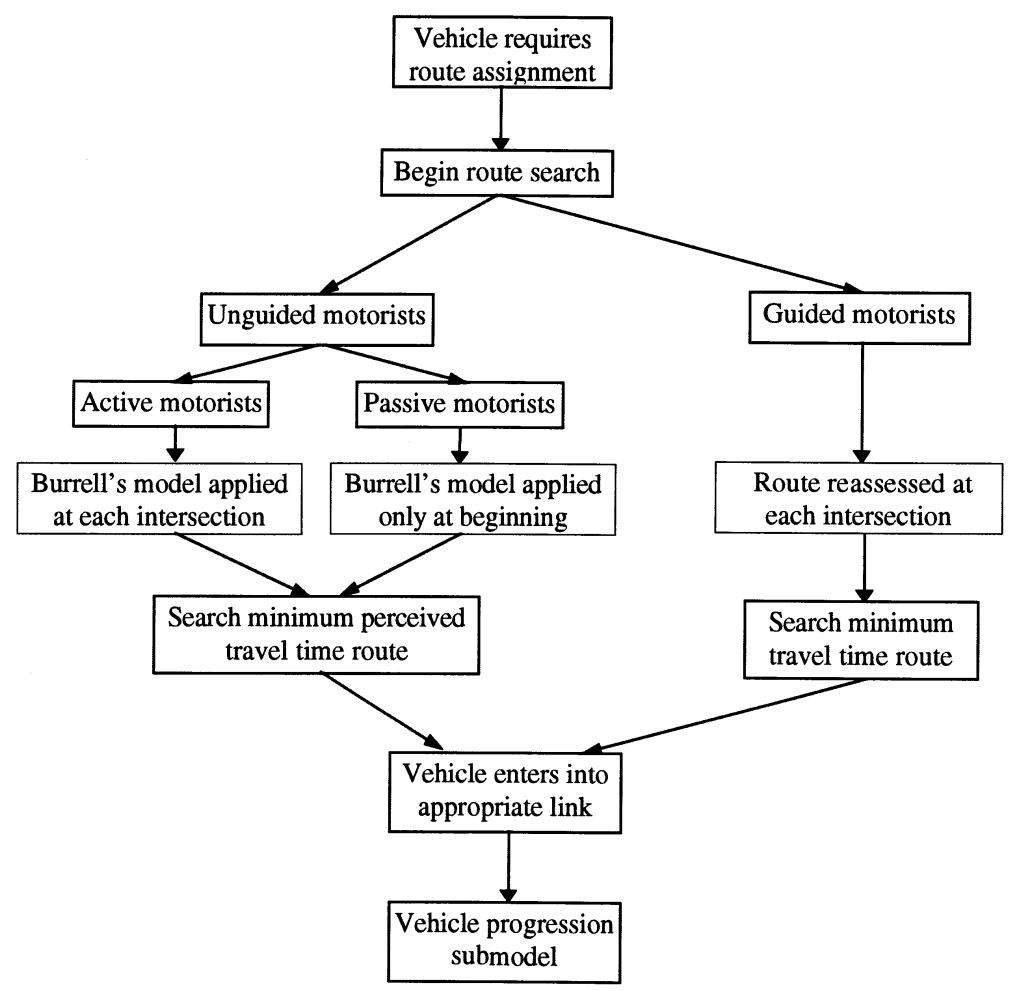

FIGURE 2 Route assignment process.

estimated average travel times on available alternative routes. Keeping these findings in mind, Burrell's model (7) has been applied to take into account the distribution of perceived travel times of unguided motorists in selecting travel routes. The following assumptions have been made in modeling route assignment of unguided motorists.

- Unguided motorists attempt to travel on minimum time (or quickest) routes.

- Unguided motorists (passive and active) choose their routes based on the perceived travel times (which may be different from actual travel times) on alternative routes.

- The perceived travel time for any link is derived by applying Burrell's (7) spread factor to the actual travel time of that link.

- The literature on Burrell's model (7) does not cater to perception distribution in turning delays at intersections; therefore, perception distribution for turning delay components has been ignored.

Figure 2 shows the application of Burrell's model to obtain perceived travel times required for estimation of the quickest routes for unguided motorists.

\section{Routes of Active Unguided Motorists}

The route selection method for active unguided motorists is the same as for passive unguided motorists. However, active unguided motorists reassess their travel routes at every intersection and modify their routes if they perceive a quicker route between the present location and the destination.

\section{Routes of Guided Motorists}

Modeling the route choice behavior of guided motorists has been a complex task. The route selection of guided motorists is quite different compared with unguided motorists. Prominent differences in characteristics of guided and unguided motorists can be outlined as follows:

1. For route selection purposes, the guided motorists receive accurate and up-to-date information about link travel times and delays at intersections from the control center, whereas unguided motorists have to figure out these components themselves by applying their knowledge about the network.

2. DRGS are often equipped with the historical traffic information that can be applied to forecast the travel times on links and turning delays at intersections at a given time of day. Therefore, DRGS recommend a route to guided motorists by considering not only the current traffic conditions but also the predicted behavior of the network during the expected journey period of motorists. For unguided motorists, however, the prediction of network behavior is not taken into account; they find their routes based on only the current performance.

3. Every time a guided motorist reaches an intersection, the alternative routes are recalculated by taking into account the current and predicted behavior of the network. The guided motorist is directed to a new route if a quicker route is found. Similarly, for active unguided motorists, routes are recalculated at intersections, but only current network conditions are considered. For passive unguided motorists, however, the routes are not recalculated during the journey.

Routes for guided motorists are calculated by a dynamic shortestpath algorithm. This method is able to compute the quickest route 
on a road network where link travel times and intersection delays change with time.

\section{Vehicle Progression Submodel}

The vehicle progression submodel manages the movement of all vehicles traversing their routes and moving toward their destinations. The movement of vehicles is performed by executing four events named start journey, reach intersection, enter link, and complete journey.

- Start journey: Each newly generated vehicle is assigned to the event "start journey." During this event, the travel route between origin and destination for the vehicle is identified, applying the appropriate route selection method for that particular vehicle type.

- Reach intersection: During this event, two tasks are performed. First, the routes for guided motorists and active unguided motorists are reassessed and motorists are diverted to a new route if a quicker route is discovered. Second, this event accounts for clearing the vehicles through the intersections. After the motorist has traversed the intersection, he arrives at the starting point of the enter link.

- Enter link: This event accounts for the movement of a particular vehicle along the link. The amount of time required to traverse the link is calculated using the link characteristics and a wellaccepted linear speed-density relationship.

- Complete journey: This covers the arrival of a vehicle at the destination and removes that vehicle from the network.

Detailed discussions on these events and scope for additional events such as road incidents, vehicle breakdowns and intermediate refuel stops have been reported elsewhere $(8,9)$.

\section{Analysis Submodel}

Objectives of the analysis submodel are twofold. First, during simulation, it estimates the performance of links and the network at user-defined regular intervals. The purpose of computing performance measures is to investigate the variation of performance of a single link, a group of links, and the network during the simulation. Second, at the end of simulation, the performance measures for motorists (mean speed, trip length, travel time, and frequency of route changes), links (mean speed, flow, and density), and network (traffic congestion and its distribution among links) are estimated by processing information stored during the simulation.

The output consists of performance analyses of motorists and network. By analyzing these data, the potential benefits of route guidance can be assessed. The performance of guided and unguided motorists can be further compared by analyzing the mean and variance of trip length and journey time.

\section{Summary of Simulation Procedures}

As described, for unguided motorists, the minimum time routes are estimated by Burrell's model (7). For guided motorists, the future traffic flows on links during their expected journey time are estimated. By using these estimates, routes for guided motorists are computed by a dynamic minimum time path algorithm.
Once routes are selected for motorists, the vehicle progression is performed. The vehicle progression submodel is the hub of the simulation model (see Figure 1) and responsible for moving vehicles along with reference to other submodels as needed. The movement of vehicles is performed with the help of four events. These events are repeatedly executed in order to navigate vehicles toward their destinations.

Along with simulating vehicle movements, the vehicle progression submodel routinely sends the control to other submodels, mainly for three reasons: (a) to generate new vehicles to match the travel demand during the simulation, $(b)$ to recalculate the routes for guided motorists and active unguided motorists as they approach intersections, and $(c)$, at predefined intervals the control is directed to the analysis submodel to estimate link and network performance measures.

The simulation starts with an empty network and allows a warm-up period before proceeding for observations on network and motorist performance. The simulation is completed when the last motorist reaches the destination and leaves an empty network. This is achieved by disabling the vehicle generation submodel after a specified duration of simulation. At the end of simulation the final analysis is performed, and performance indicators related to links, network, and different motorists are produced as the output.

\section{INFLUENCE OF INTERSECTION DELAY ON ROUTE GUIDANCE PERFORMANCE}

To demonstrate the application of the simulation model in urban road networks, the proposed model has been applied to investigate the effects of delays at intersections on the route choice behavior of motorists. This is in contrast to previous applications, which have covered a number of interesting scenarios from the planning point of view as already reported elsewhere $(6,8-10)$. Important findings of earlier investigations are:

- Travel time savings for guided motorists are large when the subscription level of dynamic route guidance is relatively small. Their savings are reduced marginally at higher levels of participation.

- Unguided motorists also benefit as a result of dynamic route guidance. Understandably, the magnitude of travel time savings for unguided motorists is low compared with guided motorists. The availability of travel time savings to both groups of motorists is seen as an important marketing advantage to the planners and developers of route guidance systems.

- Relatively high travel time savings are observed under high travel demands.

- Usage of route guidance reduces traffic congestion.

- DRGS is comparatively more effective on large and complicated road networks.

In previous applications, for simplicity, the journey times for motorists were assumed to consist only of the link travel times; delays at intersections were ignored. It has been acknowledged that in motorists' journey time the contribution of delays at intersections may be substantial in urban road networks. The influence of intersection delay on the performance of DRGS has been investigated with the objective of verifying earlier findings and developing guidelines for future research work. 
A simplistic network has been selected for this study (Figure 3). The network consists of 8 nodes and 14 links.

- Nodes 1 and 8 are the origin and the destination nodes, respectively. All motorists start their journey from node 1 and terminate at node 8 .

- Links connecting node pairs 1-2, 2-4, 4-6, 6-8, 1-3, 3-5, 5-7, and $7-8$ are each $2 \mathrm{~km}(1.24 \mathrm{mi})$ long. Interchange links connecting node pairs $2-3,4-5$, and 6-7 are $0.2 \mathrm{~km}(0.12 \mathrm{mi})$ long. Free flow speed on all links is $60 \mathrm{kph}(37.2 \mathrm{mph})$.

- Links connecting node pairs 1-2, 2-3, 6-8, and 7-8 are centroid connectors with infinite capacity. All other links have a capacity of $1,000 \mathrm{veh} / \mathrm{hr}$.

The network represents two parallel routes between origin and destination with three connector links to switch from one route to another. The length of interchange links ( $200 \mathrm{~m}$ or $219 \mathrm{yd}$ ) has been intentionally kept small to provide an easy interchange to motorists between the two parallel routes. In terms of distance and free-flow travel time, only two shortest routes are available between origin and destination and, therefore, high returns are not expected from dynamic route guidance in this example.

Applications on a variety of complex networks have been already reported elsewhere, as previously mentioned. The reason for choosing a small network with a single origin-destination pair has been to isolate and understand the influence of intersection delays on the route choice behavior of guided and unguided motorists. The network includes six intersections represented by nodes $2,3,4,5,6$, and 7. These intersections allow three possible turning movements such as right turn, through traffic, and left turn.

\section{Intersection Delay Strategy}

For the treatment of traffic movements at intersections, right-hand driving traffic conditions as observed in the United States have been assumed. Benefits of route guidance have been analyzed under three scenarios of intersection delay.

1. No delay scenario: All turning delays have been ignored. The scenario serves as a basis for comparing the influence of intersection delay on DRGS performance.

2. Constant delay scenario: Intersection delays are constant. Delay at intersections for through traffic and right-turning traffic have been assigned as 30 seconds. For left turners, delays at intersections are 45 seconds.

3. Variable delay scenario: Intersection delays vary during simulation. Intersection delays for respective turning movements increase uniformly from a designated minimum value to a maximum value.

It should be noted that, the minimum and maximum values of delay at intersections have been chosen in such a way that mean value of

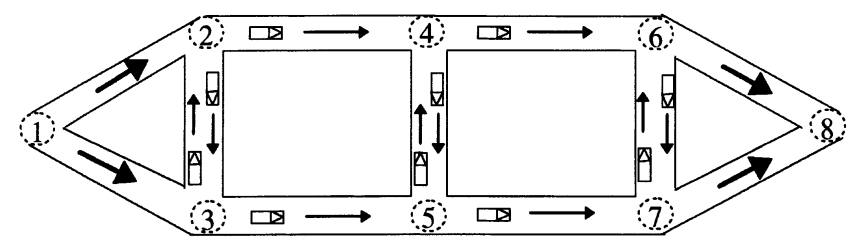

FIGURE 3 Sketch of road network. turning delay for each turning movement remains equal to the value of corresponding delay adopted in the constant delay scenario. During a one-hour simulation, the intersection delays for through traffic and right turn increase from 15 seconds to 45 seconds. Similarly, for left turns, delays increase from 30 seconds to 60 seconds.

Before analysis of intersection delay, it was anticipated that benefits of route guidance would be minimal for the no delay scenario and most substantial for the variable delay scenario because $(a)$ inclusion of turning delay adds another variable to route selection process and makes route evaluation complex, the added complexity increases chances of selecting a long route by unguided motorists, and, in these situations, the guided motorists are better positioned to achieve reductions in travel times; and $(b)$ the unguided motorists select their routes based on the present traffic conditions, and in variable delay scenario they should be worse off as their selected routes may deteriorate during the journey due to variations in intersection delays.

The travel time savings, link travel times, intersection delay, and journey speeds have been estimated to verify above hypotheses.

\section{Estimated Travel Time Savings}

Figure 4 shows savings on travel time for guided and unguided motorists. The horizontal axis denotes the proportion of guided motorists in the vehicle fleet. These travel time savings have been estimated with respect to travel times observed with no guided motorists in the traffic stream. Graphs for unguided motorists indicate a relatively flat shape under each scenario of intersection delay. For unguided motorists, the travel time savings are relatively small compared to guided motorists. These observations agree with earlier applications of this model $(6,8-10)$.

As expected, benefits of route guidance are relatively large when turning delays are included. Surprisingly, the travel time savings in variable delay scenario is smaller than corresponding savings in constant delay scenario. The largest benefits were expected in variable delay scenario as DRGS guides motorists accurately by taking into account the actual turning delays in a varying turning delay regime.

\section{Estimated Intersection Delay}

Figure 5 shows the mean intersection delays (in minutes) for guided and unguided motorists. It should be noted that intersection delays do not exist in the no delay scenario. Therefore, graphs for guided and unguided motorists in the no delay scenario correspond to the horizontal axis in Figure 5. The following observations have been made.

- The mean turning delay for guided motorists is smaller than the corresponding value for guided motorists at any given subscription level of route guidance. This happens primarily as a result of excess travel distance performed by some unguided motorists that is evident from simulation results. The guided motorists tend to travel on straight routes that minimize the turning delay for them.

- The mean turning delay is the smallest for the no delay scenario, the largest for the variable delay scenario, and intermediate for the constant delay scenario. The same order exists in travel time savings for guided and unguided motorists. This leads to proportional dependence of benefits of route guidance on intersection delays.

As described, the average turning delays at intersections for the variable delay scenario during a 1-hour simulation have been kept equal 


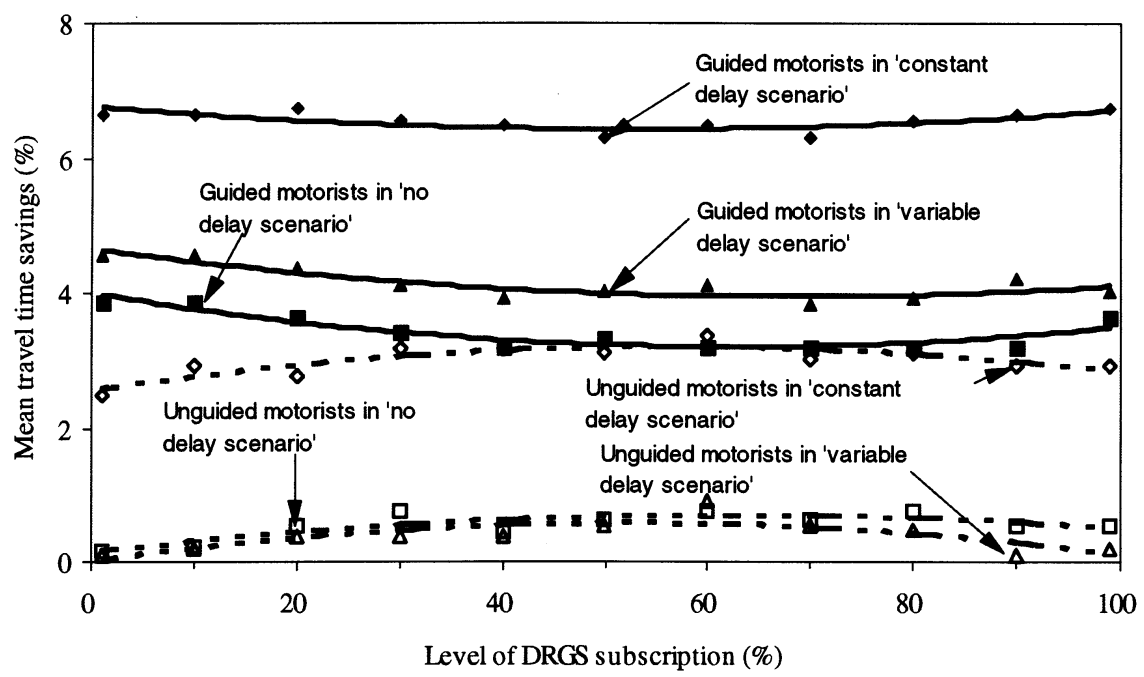

FIGURE 4 Estimated savings on travel time.

to respective turning movements in the constant delay scenario. Therefore, it was expected that mean values of turning delay in both scenarios should be close; but results show a consistent difference on simulated turning delays in the two scenarios. This finding needs to be further investigated.

In variable delay scenario, in the second half of the simulation when turning delays are substantial, the chances of unguided motorists following long routes are reduced as turning delays contribute a significant amount in journey time. This yields reduced mean delays under variable delay scenarios. Detailed analysis of route choice behavior of motorists in the three considered scenarios have been performed to explain reasons for low benefits in the variable delay scenario.

\section{Analytical Perspective of Intersection Delay on Route Selection}

To analyze the influence of intersection delay in further detail, the penalties of adopting a long route by making an incorrect turn have been investigated in five situations: $(a)$ no delay scenario, $(b)$ constant delay scenario, $(c)$ beginning of simulation in variable delay scenario, $(d)$ middle of simulation in variable delay scenario, and (e) end of simulation in variable delay scenario.

In the network shown in Figure 3, the minimum extra delay of an incorrect turn would be the travel time on a connector link $(200 \mathrm{~m}$ or 219 yd long) plus delay for a left turn movement. Free-flow traffic conditions on interchange links can be assumed as the majority of traffic tends to travel on straight routes. Under free-flow traffic conditions, the travel time of a connector link is

Average link travel time $=$ length of link/free-flow speed

Average penalty (in sec) for an incorrect turn by unguided motorists in any scenario is

Average link travel time + mean left turning delay

In percentage terms, penalties for choosing an incorrect route in the above five situations increase journey times:

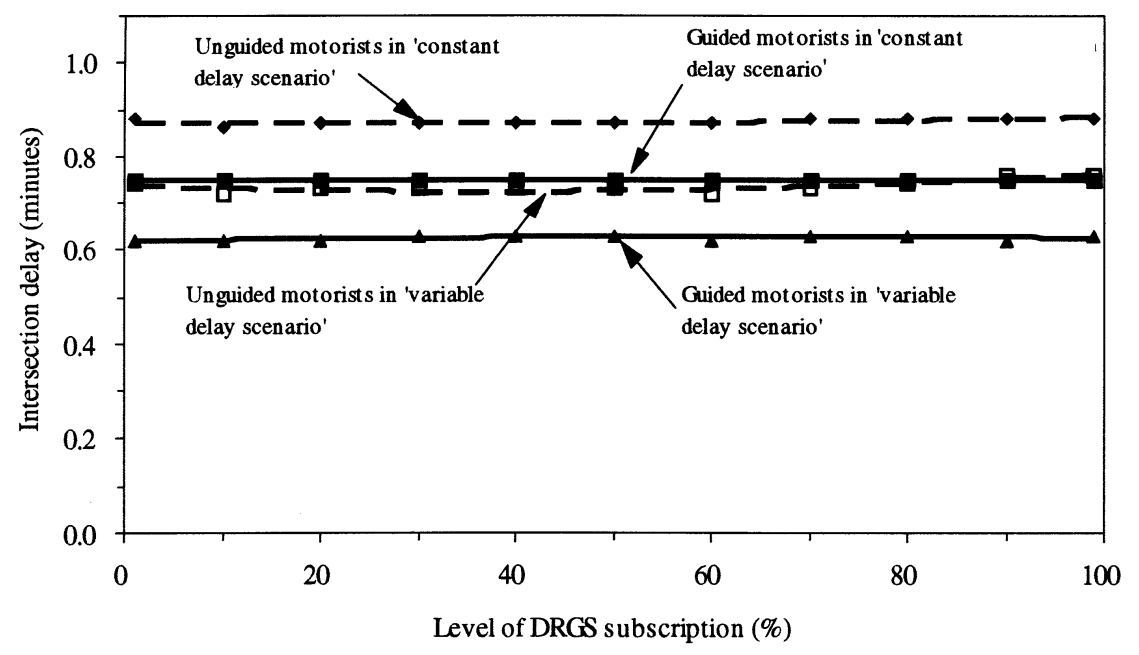

FIGURE 5 Intersection delay for motorists. 
Percent increase in journey time $=100 \times$ penalty/mean journey time

Proportion of motorists traveling on long routes can be determined by the following formula:

Proportion $=$ travel time savings/percent increase in journey time .

Estimated proportions of motorists selecting nonoptimum routes have been shown in Table 1. It is clear that in the no delay scenario about 181 percent of motorists make an incorrect turn. Similarly, in constant delay scenario, about 52 percent of motorists travel on long routes. In variable delay scenario, the proportion of unguided motorists traveling on inefficient routes changes during the simulation because of variations in turning delay. The proportion for inefficient unguided motorists in this scenario has been identified as 170 , 81 , and 64 percent at the beginning, middle, and end of the simulation period, respectively. Unrealistic large values of 181 percent for no delay scenario and 110 percent for variable delay scenario are due to motorists who make more than one incorrect turn.

These observations indicate that motorists are less likely to adopt long routes if penalties are large. Obviously in these situations, benefits of route guidance will not be substantial. Therefore, benefits of route guidance do not necessarily increase with delay at intersections. It has been speculated that there is an optimum combination of turning delay at which route guidance will provide the largest benefit. This phenomenon needs to be further investigated by applying the simulation model on constant delay scenario with different values of turning delay.

To further support these findings analysis of link travel speeds has also been performed.

\section{Estimated Link Travel Speeds}

Link travel speeds for guided motorists are shown in Figure 6. Link travel times and, subsequently, link travel speeds have been estimated by subtracting turning delays from journey times.

The following observations have been made:

1. Shapes of graphs for the three scenarios imply that mean link travel speed is insensitive to the proportion of guided motorists. This finding agrees with patterns observed in earlier studies.
2. Proportionally large link travel speeds have been observed in the no delay scenario. This scenario represents the uninterrupted traffic-flow conditions with no interchange delay. It is obvious that high travel speeds will be observed in such conditions.

3. Mean link speeds for constant delay and variable delay scenarios are small compared to the no delay scenario. The reduction in travel speed primarily occurs because of reduction in the performance of links as a result of vehicles waiting at intersections. This finding can be confirmed by comparing magnitudes of mean intersection delays in constant delay and variable delay scenarios. Mean intersection delays in constant delay scenarios are larger than the delays in variable intersection scenarios, resulting in better performance of links under constant delay scenarios.

4. The free-flow speed on all links has been assigned as $60 \mathrm{kph}$ (37.2 $\mathrm{mph}$ ). The mean speed closer to free-flow speed as observed in constant delay scenario represents minimum congestion in traffic similarly; low mean speed in variable delay scenario represents relatively congested flow of traffic. When these observations were compared with the observations on travel time savings, it was concluded that benefits of route guidance are greater in congested traffic conditions. This finding supports the general expectation from the route guidance and agrees well with investigations and expectations reported elsewhere (11).

In brief, the observations made on the three scenarios considered with the objective of understanding the influence of turning delay on performance of DRGS can be outlined as

- The presence of turning delay results in increasing the total benefits from dynamic route guidance, as expected.

- The amount of turning delay at intersections significantly affects the route choice behavior of motorists.

- Benefits of application of DRGS increase with the increase in mean intersection delay. There could be an optimum intersection delay strategy at which DRGS may reap maximum benefits.

\section{CONCLUSIONS}

The evaluation model of a generic dynamic route guidance project has been discussed. Proposed methodology allows quantification of travel time savings for various types of motorists in the traffic

TABLE 1 Penalty of Incorrect Turning Movement in Considered Situations

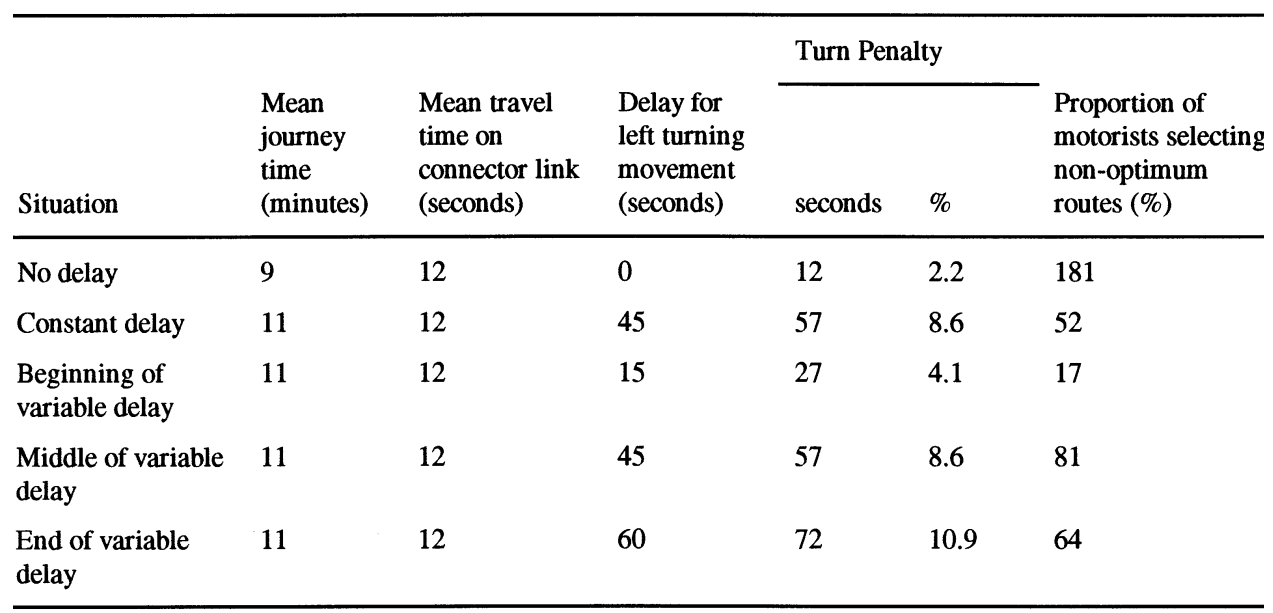




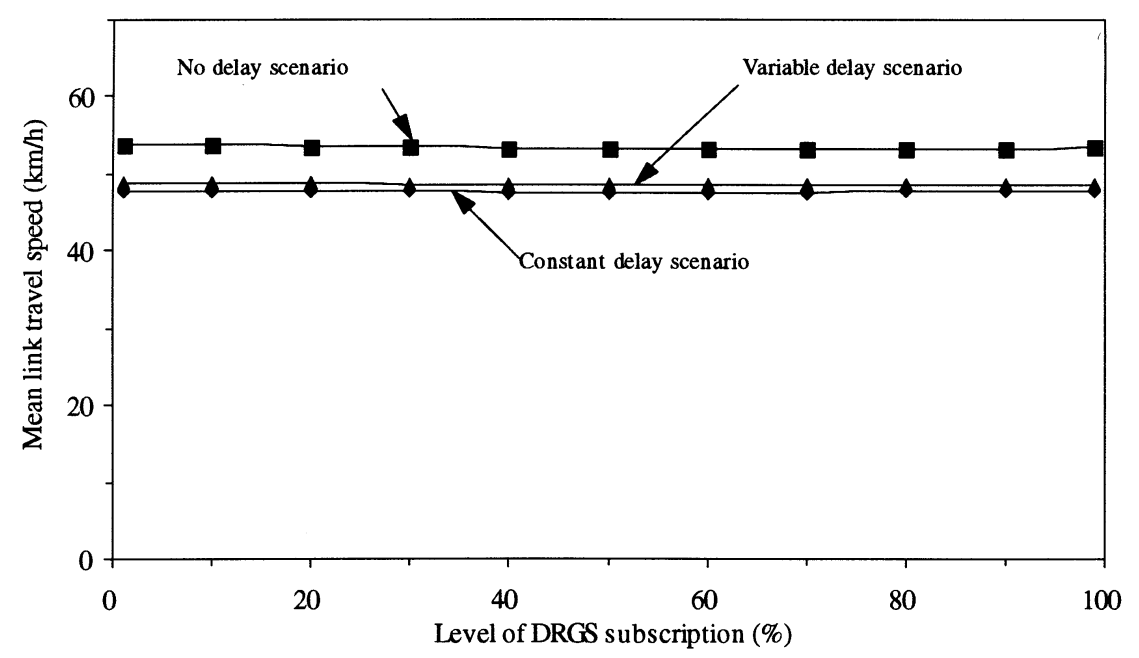

FIGURE 6 Link travel speed for guided motorists.

stream as a result of route guidance. The evaluation model consists of four submodels: vehicle generation, vehicle progression, route selection, and analysis. The methodology allows the analysis of benefits of route guidance by estimating the performance of guided and unguided motorists and the performance of the road network. The impact of route guidance on the route selection behavior of different motorists can also be studied using this model.

As a case study application, the evaluation model has been applied to investigate the influence of turning delays on the performance of DRGS. On a simple road network of 8 nodes and 14 links, the performance of DRGS has been investigated under three turning delay scenarios referred to as no turning delay, constant turning delay, and varying turning delay. Investigations of these scenarios indicate that the presence of turning delays provides an incentive to introduce route guidance. As expected, benefits of route guidance are minimal under the scenario of no delay at intersections. High travel time savings have been achieved under the constant turning delay scenario.

Findings also suggest that route guidance concept benefits both guided and unguided motorists. Inclusion of turning delays in analysis makes DRGS more productive. Therefore, road systems with intersection delays such as urban networks are prime targets for route guidance proposals.

\section{REFERENCES}

1. Armstrong, B. D. The Need for Route Guidance. Transport Road Research Laboratory Report, SR 330, Crowthorne, U.K., 1977.

2. Jeffery, D. J. The Potential Benefits of Route Guidance. Transport Road Research Laboratory Report, LR 997, Crowthorne, U.K., 1981.
3. Robb, M. C. Route Information System for Motorists. Transport Reviews, Vol. 7, No. 3, 1987, pp. 259-276.

4. King, G. F., and T. M. Mast. Excess Travel: Causes, Extent and Consequences. In Transportation Research, Record 1111, TRB, National Research Council, Washington, D.C., 1987, pp. 126-134.

5. Bovy, P. H. L., and E. Stern. Route Choice: Wayfinding in Urban Networks. Studies in Operational Regional Science, Vol. 9, Kluwer Academic Publishers, The Netherlands, 1990.

6. Upadhyay, P. K., U. Vandebona, and P. Hidas. Performance of DRGS in Different Size Networks. In Proceedings of the 19th Australasian Transport Research Forum (ATRF), Lorne, Victoria, Australia, Vol. 19, Sept. 1994, pp. 101-115.

7. Burrell, J. E. Multipath Route Assignment and Its Application to Capacity Restraint. In Proceedings of the 4th International Symposium on the Theory of Traffic Flow, June 1968, pp. 210-219.

8. Upadhyay, P. K., U. Vandebona, and P. Hidas. Performance Evaluation Methodology for Route Guidance Systems. In Proceedings of the International Conference on Advances in Strategic Technologies (ICAST'95), Bangi, Malaysia, July 1995, pp. 155-164.

9. Upadhyay, P. K., U. Vandebona, and P. Hidas. Simulation Modeling of Route Guidance Systems. In Proceedings of the International Association of Science and Technology for Development (JASTED) Conference, Gold Coast, Australia, May 1996, CD-ROM index 242-274, 9 p.

10. Upadhyay, P. K., U. Vandebona, and P. Hidas. An Evaluation Methodology for Dynamic Route Guidance Systems. In Proceedings of the 17th Australian Road Research Board (ARRB) Conference, Gold Coast, Australia, Aug. 1994, Vol. 17:4, 1994, pp. 213-229.

11. Upadhyay, P. K., P. Hidas, and U. Vandebona. Route Guidance as a Congestion Management Tool. In Proceedings of the 27th International Symposium on Advanced Transportation Application (ISATA), Aachen, Germany, Nov. 1994, pp. 51-58.

Publication of this paper sponsored by Committee on User Information Systems. 